\title{
On the exponent of distribution of the ternary divisor function
}

\section{Journal Article}

\section{Author(s):}

Fouvry, Étienne; Kowalski, Emmanuel; Michel, Philippe

Publication date:

2015-01

Permanent link:

https://doi.org/10.3929/ethz-b-000084579

Rights / license:

In Copyright - Non-Commercial Use Permitted

\section{Originally published in:}

Mathematika 61(1), https://doi.org/10.1112/S0025579314000096 


\title{
ON THE EXPONENT OF DISTRIBUTION OF THE TERNARY DIVISOR FUNCTION
}

\author{
ÉTIENNE FOUVRY, EMMANUEL KOWALSKI AND PHILIPPE MICHEL
}

Abstract. We show that the exponent of distribution of the ternary divisor function $d_{3}$ in arithmetic progressions to prime moduli is at least $1 / 2+1 / 46$, improving results of Friedlander-Iwaniec and Heath-Brown. Furthermore, when averaging over a fixed residue class, we prove that this exponent is increased to $1 / 2+1 / 34$.

$\S 1$. Introduction and statement of the main results. For any positive integer $k \geqslant 1$, we denote by $d_{k}$ the $k$-fold divisor function: for $n$ a positive integer, $d_{k}(n)$ is the number of solutions of the equation

$$
n=n_{1} \ldots n_{k},
$$

where the $n_{i}$ are positive integers. The purpose of this paper is to investigate the exponent of distribution of the ternary divisor function $d_{3}$ in arithmetic progressions. More generally, we will say that a real number $\Theta>0$ is an exponent of distribution for $d_{k}$ restricted to a set $\mathcal{Q}$ of moduli if, for any $\varepsilon>0$, for any $q \in \mathcal{Q}$ with $q \leqslant x^{\Theta-\varepsilon}$ and any residue class $a \bmod q$ with $(a, q)=1$, we have a uniform asymptotic formula

$$
\sum_{\substack{n \equiv a \bmod q \\ n \leqslant x}} d_{k}(n)=\frac{1}{\varphi(q)} \sum_{\substack{(n, q)=1 \\ n \leqslant x}} d_{k}(n)+O\left(\frac{x}{q(\log x)^{A}}\right)
$$

for any $A>0$ and $x \geqslant 2$, the implied constant depending on $A$ and $\varepsilon$ only. If $\mathcal{Q}$ contains all positive integers, we speak only of exponent of distribution.

It is widely believed $\Theta=1$ is an exponent of distribution for all $k$. This fact, if true, has deep consequences on our understanding of the distribution of primes in arithmetic progressions to very large moduli, going beyond the direct reach of the generalized Riemann hypothesis. It is therefore not surprising that this problem has been studied extensively, and that it is especially relevant to obtain an exponent of distribution $\Theta>1 / 2$, since this goes beyond the techniques involving the Bombieri-Vinogradov theorem.

As a consequence of the combinatorial structure of $d_{k}$ (essentially by Dirichlet's hyperbola method in dimension $k$ ), one instantly deduces that 
$\Theta=1 / k$ is an exponent of distribution for $d_{k}$, in particular $\Theta=1$ for $k=1$. It was noted by Linnik and Selberg that for $k=2$ (the classical divisor function), a fairly direct application of Weil's bound for Kloosterman sums yields $\Theta=2 / 3$.

The only other case for which an exponent of distribution greater than $1 / 2$ is known is for $d_{3}$ : in their groundbreaking paper, Friedlander and Iwaniec [9] showed that $\Theta=1 / 2+1 / 230$ is an exponent of distribution, a value later improved by Heath-Brown to $\Theta=1 / 2+1 / 82$ [10]. The proof of these two results uses deep applications of Deligne's proof of the Riemann hypothesis for algebraic varieties over finite fields. Our main result is a further, rather significant, improvement in the case of prime moduli.

THEOREM 1.1. For every non-zero integer a, every $\varepsilon, A>0$, every $x \geqslant 2$ and every prime $q$, coprime with $a$, satisfying

$$
q \leqslant x^{1 / 2+1 / 46-\varepsilon}
$$

we have

$$
\sum_{\substack{n \equiv a \bmod q \\ n \leqslant x}} d_{3}(n)=\frac{1}{\varphi(q)} \sum_{\substack{(n, q)=1 \\ n \leqslant x}} d_{3}(n)+O\left(\frac{x}{q(\log x)^{A}}\right),
$$

where the implied constant only depends on $\varepsilon$ and $A$ (and not on a); in other words, the value $\Theta=1 / 2+1 / 46$ is an exponent of distribution for the divisor function $d_{3}$ restricted to prime moduli.

It is certainly possible to extend our arguments to composite moduli. This would require some generalization of our main tools, which are general estimates for sums of trace functions over finite fields twisted by Fourier coefficients of Eisenstein series (see Theorem 3.1 below).

1.1. Distribution on average. In applications, estimates like (1.1) are often required only on average over moduli $q \leqslant Q$ and it is no surprise that sometimes these become available for $Q=x^{\theta}$ and $\theta$ larger than the known exponents of distribution. For instance, since the function $d_{k}$ is multiplicative, the large sieve inequality implies that (1.1) holds on average for any $\theta<1 / 2$ (see, e.g., [12] or [15]). Concerning $d_{3}$, Heath-Brown [10, Theorem 2] proved the following result (in a slightly stronger form):

$$
\sum_{q \leqslant Q} \max _{y \leqslant x} \max _{(a, q)=1}\left|\sum_{\substack{n \equiv a \bmod q \\ n \leqslant y}} d_{3}(n)-\frac{1}{\varphi(q)} \sum_{\substack{(n, q)=1 \\ n \leqslant y}} d_{3}(n)\right|=O\left(x^{40 / 51+\varepsilon} Q^{7 / 17}\right),
$$

which shows that (1.1) holds on average for $q \leqslant x^{11 / 21-\varepsilon}$.

Although we can not improve this (on average over prime moduli), we are able to improve Theorem 1.1 for $d_{3}$ on average over prime moduli in a single residue class $n \equiv a(\bmod q)$, where $a \neq 0$ is fixed. 
THEOREM 1.2. For every non-zero integer $a$, for every $\varepsilon>0$ and for every $A>0$, we have

$$
\sum_{\substack{q \leqslant x^{9 / 17-\varepsilon} \\ q \text { prime } q \nmid a}}\left|\sum_{\substack{n \equiv a \bmod q \\ n \leqslant x}} d_{3}(n)-\frac{1}{\varphi(q)} \sum_{\substack{(n, q)=1 \\ n \leqslant x}} d_{3}(n)\right|=O\left(\frac{x}{(\log x)^{A}}\right),
$$

where the implied constant only depends on $(a, A, \varepsilon)$.

Remark 1.3. It is implicit from our proof and from the results of [1] on which it is based that this estimate holds uniformly for $1 \leqslant|a| \leqslant x^{\delta}$, for some $\delta>0$ depending on $\varepsilon$.

1.2. Remarks on the proofs. The proof of Theorem 1.1 builds on two main ingredients developed in [7] and [8].

(1) A systematic exploitation of the spectral theory of modular forms; for instance, although our most important estimate involves only the divisor function, its proof passes through the full spectrum of the congruence subgroup $\Gamma_{0}(q) \subset \mathrm{SL}_{2}(\mathbb{Z})$.

(2) The formalism of Frobenius trace functions modulo a prime, like the normalized Kloosterman sums $a \mapsto p^{-1 / 2} S(a, 1 ; p)$ : such functions are considered as fundamental building blocks in estimates, and not necessarily "opened" too quickly as exponential sums (for instance, the crucial estimate of a three-variable character sum in [9] is, in our treatment, hidden in the very general statement of Theorem 3.2, which follows from [8]).

The outcomes are two different estimates (Theorems 3.1 and 3.2) which are applied through a simple combinatorial decomposition of the main sum (compare, e.g., \$5.3 with [10, §7]).

The proof of Theorem 1.2 combines these estimates with the "Kloostermaniac" techniques pioneered by Deshouillers and Iwaniec and pursued with great success by Bombieri, Fouvry, Friedlander and Iwaniec to study primes in large arithmetic progressions.

Remark 1.4. After the first version of this paper had been submitted for publication, the arithmetic importance of the exponent of distribution of the ternary divisor function for suitable large moduli was highlighted again in Zhang's groundbreaking work [16] on bounded gap between primes. Some of the techniques developed in the present paper have since been used, within the project Polymath 8, to give improvements of Zhang's results (see [13, §9] for a discussion).

1.3. Notation. We denote $\mathrm{e}(z)=\mathrm{e}^{2 \mathrm{i} \pi z}$ for $z \in \mathbb{C}$. For $n \geqslant 1$ and for an integrable function $w: \mathbb{R}^{n} \rightarrow \mathbb{C}$, we denote by

$$
\widehat{w}(\xi)=\int_{\mathbb{R}^{n}} w(t) \mathrm{e}(-\langle t, \xi\rangle) d t
$$

its Fourier transform, where $\langle\cdot, \cdot\rangle$ is the standard inner product on $\mathbb{R}^{n}$. 
If $q \geqslant 1$ is a positive integer and if $K: \mathbb{Z} \longrightarrow \mathbb{C}$ is a periodic function with period $q$, its Fourier transform is the periodic function $\widehat{K}$ of period $q$ defined on $\mathbb{Z}$ by

$$
\widehat{K}(n)=\frac{1}{\sqrt{q}} \sum_{h \bmod q} K(h) \mathrm{e}\left(\frac{h n}{q}\right)
$$

(note the minor inconsistency of sign choices). We have $\widehat{\widehat{K}}(n)=K(-n)$ for all integers $n$.

Given a prime $p$ and a residue class $a$ invertible modulo $p$, we denote by $\bar{a}$ the inverse of $a$ modulo $p$. For a prime $p$ and an integer $a$, the normalized hyper-Kloosterman $\operatorname{sum~}_{\mathrm{Kl}_{k}}(a ; p)$ is given by

$$
\mathrm{Kl}_{k}(a ; p):=\frac{1}{p^{(k-1) / 2}} \sum_{\substack{x_{1}, \ldots, x_{k} \\ x_{1} \cdots x_{k} \equiv a \bmod p}} \mathrm{e}\left(\frac{x_{1}+\cdots+x_{k}}{p}\right) .
$$

The notation $q \sim Q$ means $Q<q \leqslant 2 Q$, and $f(x)=O(g(x))$ for $x \in X$ is synonymous with $f(x) \ll g(x)$ for $x \in X$.

\section{§2. Summation formulas.}

2.1. Poisson summation formula. We recall a form of the Poisson summation formula in arithmetic progressions.

Lemma 2.1. For any positive integer $q \geqslant 1$, any function $K$ defined on integers and q-periodic, and any smooth function $V$ compactly supported on $\mathbb{R}$, we have

$$
\sum_{n \geqslant 1} K(n) V(n)=\frac{1}{\sqrt{q}} \sum_{m} \widehat{K}(m) \hat{V}\left(\frac{m}{q}\right),
$$

and in particular

$$
\sum_{n \equiv a \bmod q} V(n)=\frac{1}{q} \sum_{m} \mathrm{e}\left(\frac{a m}{q}\right) \hat{V}\left(\frac{m}{q}\right) .
$$

2.2. The tempered Voronoi summation formula. We will also make crucial use of a general (soft) version of the classical summation formula of Voronoi for the divisor function $d_{2}$, which goes back to Deshouillers and Iwaniec [3, Lemma 9.2]. This formula is called the tempered Voronoi summation formula in [11, Proposition 4.11], and amounts essentially to an application of the Poisson formula in two variables $(x, y)$ to a function depending on the product $x y$.

We define the Voronoi transform $\breve{K}$ of a $p$-periodic function $K: \mathbb{Z} \longrightarrow \mathbb{C}$ by

$$
\breve{K}(n)=\frac{1}{\sqrt{p}} \sum_{\substack{h \bmod p \\(h, p)=1}} \hat{K}(h) \mathrm{e}\left(\frac{n \bar{h}}{p}\right) .
$$


In other words, we have

$$
\check{K}(n)= \begin{cases}\frac{1}{\sqrt{p}} \sum_{h_{1} h_{2}=n} \widehat{K}\left(h_{1}\right) \mathrm{e}\left(\frac{h_{2}}{p}\right), & \text { if } p \nmid n, \\ K(0)-\frac{\widehat{K}(0)}{\sqrt{p}}, & \text { if } p \mid n .\end{cases}
$$

Proposition 2.2 (Tempered Voronoi formula modulo primes). Let $p$ be a prime number, let $K: \mathbb{Z} \longrightarrow \mathbb{C}$ be a p-periodic function, and let $G$ be a smooth function on $\mathbb{R}^{2}$ with compact support. We have

$$
\sum_{m, n \in \mathbb{Z}} K(m n) G(m, n)=\frac{\widehat{K}(0)}{\sqrt{p}} \sum_{m, n \in \mathbb{Z}} G(m, n)+\frac{1}{p} \sum_{m, n} \check{K}(m n) \widehat{G}\left(\frac{m}{p}, \frac{n}{p}\right) .
$$

Proof. We have the discrete inversion formula

$$
K(m n)=\frac{1}{\sqrt{p}} \widehat{K}(0)+\frac{1}{\sqrt{p}} \sum_{(h, p)=1} \widehat{K}(h) \mathrm{e}\left(-\frac{h m n}{p}\right),
$$

and then for any integer $a$ coprime to $p$, the tempered Voronoi formula of [11, Proposition 4.11] gives

$$
\sum_{m, n \in \mathbb{Z}} G(m, n) \mathrm{e}\left(\frac{a m n}{p}\right)=\frac{1}{p} \sum_{m, n \in \mathbb{Z}} \widehat{G}\left(\frac{m}{p}, \frac{n}{p}\right) \mathrm{e}\left(-\frac{\bar{a} m n}{p}\right),
$$

so that the result follows by multiplying this by $\widehat{K}(-a)$ and summing over $(a, p)=1$.

2.3. The combined formula. We now combine the Poisson formula and the Voronoi formula to give a general transformation formula for three-variable sums.

COROLlary 2.3 (Poisson-Voronoi formula). Let $\boldsymbol{V}=\left(V_{1}, V_{2}, V_{3}\right)$ where $V_{i}$ are smooth functions with compact support in $] 0,+\infty[$. Let $p$ be a prime number, and let $K$ be a p-periodic function on $\mathbb{Z}$, supported on integers coprime to $p$. Define

$$
S(\boldsymbol{V} ; p, K)=\sum_{m_{1}, m_{2}, m_{3} \geqslant 1} V_{1}\left(m_{1}\right) V_{2}\left(m_{2}\right) V_{3}\left(m_{3}\right) K\left(m_{1} m_{2} m_{3}\right) .
$$

We then have

$$
S(\boldsymbol{V} ; p, K)=\mathrm{A}+\mathrm{B}+\mathrm{C}+\mathrm{D}
$$

where

$$
\mathrm{A}=\frac{\widehat{K}(0)}{\sqrt{p}} \sum \sum_{\substack{p \nmid m_{1} m_{2} \\ m_{3} \geqslant 1}} \sum V_{1}\left(m_{1}\right) V_{2}\left(m_{2}\right) V_{3}\left(m_{3}\right),
$$




$$
\begin{aligned}
\mathrm{B}= & -\frac{\widehat{K}(0)}{p^{3 / 2}} \sum_{\substack{m_{1}, m_{2} \geqslant 1 \\
p \nmid n_{3}}} \sum V_{1}\left(m_{1}\right) V_{2}\left(m_{2}\right) \widehat{V}_{3}\left(\frac{n_{3}}{p}\right) \\
\mathrm{C}= & \frac{\widehat{K}(0)}{p^{2}}\left\{\widehat{V}_{1}(0) \sum_{n_{2}} \widehat{V}_{2}\left(\frac{n_{2}}{p}\right)+\widehat{V}_{2}(0) \sum_{n_{1}} \widehat{V}_{1}\left(\frac{n_{1}}{p}\right)-\widehat{V}_{1}(0) \widehat{V}_{2}(0)\right\} \\
& \times \sum_{p \nmid n_{3}} \widehat{V}_{3}\left(\frac{n_{3}}{p}\right) \\
\mathrm{D}= & \frac{1}{p^{3 / 2}} \sum_{\substack{n_{1} n_{2} \neq 0 \\
p \nmid n_{3}}} \sum_{\hat{V}_{1}\left(\frac{n_{1}}{p}\right) \widehat{V}_{2}\left(\frac{n_{2}}{p}\right) \widehat{V}_{3}\left(\frac{n_{3}}{p}\right) \widetilde{K}\left(n_{1} n_{2}, n_{3}\right),}
\end{aligned}
$$

with

$$
\widetilde{K}(x, n)=\frac{1}{\sqrt{p}} \sum_{y \in \mathbb{F}_{p}^{\times}} \widehat{K}(n \bar{y}) \mathrm{Kl}_{2}(x y ; p) .
$$

In the sums above, $m_{1}, m_{2}, m_{3}$ run over integers $\geqslant 1$, with additional conditions, while $n_{1}, n_{2}, n_{3}$ run over all integers in $\mathbb{Z}$.

Proof. We start by applying the Poisson formula (Lemma 2.1) to the variable $m_{3}$. Denoting by $n_{3} \in \mathbb{Z}$ the dual Fourier variable, we isolate the terms with $n_{3} \equiv 0 \bmod p$ and obtain

$$
\begin{aligned}
S(\boldsymbol{V} ; p, a)= & \frac{1}{\sqrt{p}} \sum_{\substack{\left(m_{1} m_{2}, p\right)=1 \\
n_{3} \equiv 0 \bmod p}} \sum_{1} V_{1}\left(m_{1}\right) V_{2}\left(m_{2}\right) \widehat{V}_{3}\left(\frac{n_{3}}{p}\right) \widehat{K}(0) \\
& +\frac{1}{\sqrt{p}} \sum_{\substack{\left(m_{1} m_{2}, p\right)=1 \\
\left(n_{3}, p\right)=1}} \sum_{1} V_{1}\left(m_{1}\right) V_{2}\left(m_{2}\right) \widehat{V}_{3}\left(\frac{n_{3}}{p}\right) \widehat{K}\left(n_{3} \overline{m_{1} m_{2}}\right),
\end{aligned}
$$

where $\overline{m_{1} m_{2}}$ is the multiplicative inverse of $m_{1} m_{2}$ modulo $p$.

We use the Poisson formula again to transform backward the first sum, and get

$$
\sum_{n_{3} \equiv 0 \bmod p} \widehat{V}_{3}\left(\frac{n_{3}}{p}\right)=\sum_{n_{3}} \widehat{V}_{3}\left(n_{3}\right)=\sum_{m_{3}} V_{3}\left(m_{3}\right)
$$

so that this first term is equal to the quantity $A$ of the statement.

We now consider the second sum, which we denote $\Sigma(\boldsymbol{V} ; p, K)$. We apply the tempered Voronoi summation formula of Proposition 2.2 to the sum over $m_{1}$ and $m_{2}$, and to the function

$$
m \mapsto L\left(m, n_{3}\right)=\widehat{K}\left(n_{3} \bar{m}\right) \quad \text { for } p \nmid m,
$$

extended by 0 to the $m$ divisible by $p$. Denoting by $\widehat{L}$ and $\breve{L}$ the corresponding transforms with respect to the variable $m$ when $n_{3}$ is fixed, we note that 
THE EXPONENT OF DISTRIBUTION OF $d_{3}$

$$
\widehat{L}\left(0, n_{3}\right)=\frac{1}{\sqrt{p}} \sum_{x \in \mathbb{F}_{p}^{\times}} \widehat{K}(x)=-\frac{1}{\sqrt{p}} \widehat{K}(0)
$$

for every $n_{3}$ coprime to $p$, since $K(0)=0$ by assumption.

Thus we obtain

$$
\Sigma(\boldsymbol{V} ; p, K)=\Sigma_{1}(\boldsymbol{V} ; p, K)+\Sigma_{2}(\boldsymbol{V} ; p, K)
$$

where

$$
\Sigma_{1}(\boldsymbol{V} ; p, K)=-\frac{\widehat{K}(0)}{p^{3 / 2}} \sum_{\substack{m_{1}, m_{2} \geqslant 1 \\ p \nmid n_{3}}} \sum_{\substack{1 \\ n_{1}}} V_{1}\left(m_{1}\right) V_{2}\left(m_{2}\right) \widehat{V}_{3}\left(\frac{n_{3}}{p}\right)=\mathrm{B},
$$

and

$$
\begin{aligned}
\Sigma_{2}(\boldsymbol{V} ; p, K)= & \frac{1}{p^{3 / 2}} \sum_{p \nmid n_{3}} \widehat{V}_{3}\left(\frac{n_{3}}{p}\right)\left\{\breve{L}\left(0, n_{3}\right) \sum_{n_{1} n_{2}=0} \sum_{V_{1}}\left(\frac{n_{1}}{p}\right) \widehat{V}_{2}\left(\frac{n_{2}}{p}\right)\right. \\
& +\sum_{n_{1} n_{2} \neq 0} \sum_{\widehat{V}_{1}}\left(\frac{n_{1}}{p}\right) \widehat{V}_{2}\left(\frac{n_{2}}{p}\right) \breve{L}_{\left.\left(n_{1} n_{2}, n_{3}\right)\right\} .}
\end{aligned}
$$

A straightforward computation shows that

$$
\breve{L}\left(x, n_{3}\right)=\frac{1}{\sqrt{p}} \sum_{y \in \mathbb{F}_{p}^{\times}} \widehat{K}\left(n_{3} \bar{y}\right) \mathrm{Kl}_{2}(x y ; p)=\widetilde{K}\left(x, n_{3}\right) .
$$

In particular, we have

$$
\breve{L}\left(0, n_{3}\right)=-\frac{1}{\sqrt{p}} \sum_{y \in \mathbb{F}_{p}^{\times}} \widehat{K}(y)=-\widehat{L}\left(0, n_{3}\right),
$$

so, by (2.2), the first term in $\Sigma_{2}(V ; p, K)$ is

$$
\begin{aligned}
& \frac{\widehat{K}(0)}{p^{2}}\left\{\widehat{V}_{1}(0) \sum_{n_{2}} \widehat{V}_{2}\left(\frac{n_{2}}{p}\right)+\widehat{V}_{2}(0) \sum_{n_{1}} \widehat{V}_{1}\left(\frac{n_{1}}{p}\right)-\widehat{V}_{1}(0) \widehat{V}_{2}(0)\right\} \\
& \quad \times \sum_{p \nmid n_{3}} \widehat{V}_{3}\left(\frac{n_{3}}{p}\right)=\mathrm{C},
\end{aligned}
$$

while the remaining contribution is the quantity $D$.

In this paper, we will only need the following case of these transformations.

LEMMA 2.4. Let $p$ be a prime, let a be an invertible residue class modulo $p$, and let, for $n$ an integer,

$$
K(n)=\delta_{a}(n) .
$$

Then, for every $n$ not divisible by $p$ and for every $x$, we have the equality

$$
\widetilde{K}(x, n)=\frac{1}{\sqrt{p}} \mathrm{Kl}_{3}(\text { an } x ; p) .
$$


Proof. Obviously, we have $\widehat{K}(x)=(1 / \sqrt{p}) \mathrm{e}(a x / p)$, and the result then follows from the definition (2.1) after opening the Kloosterman sum.

§3. Results on trace functions. The key new input to the present paper is the application of a special case of the following very general theorem concerning algebraic trace functions summed against the divisor functions.

THEOREM 3.1 (Divisor twists of trace functions). Let $p$ be a prime number, and let $K$ be the trace function of an $\ell$-adic middle-extension sheaf $\mathcal{F}$, pointwise of weight 0 , on the affine line over $\mathbb{F}_{p}$. Assume that $\mathcal{F}$ is geometrically irreducible and is not geometrically isomorphic to an Artin-Schreier sheaf associated to an additive character modulo $p$.

Let $Q \geqslant 1$ and let $V, W$ be smooth test functions, compactly supported in $[1 / 2,2]$, such that, for $\xi>0$, one has

$$
\xi^{j} V^{(j)}(\xi), \xi^{j} W^{(j)}(\xi) \ll Q^{j},
$$

for all integers $j \geqslant 0$, with implicit constants that depend on $j$. For any $M_{1}$, $M_{2} \geqslant 1$, we have

$$
\sum_{m_{1}, m_{2} \geqslant 1} K\left(m_{1} m_{2}\right) V\left(\frac{m_{1}}{M_{1}}\right) W\left(\frac{m_{2}}{M_{2}}\right) \ll Q M_{1} M_{2}\left(1+\frac{p}{M_{1} M_{2}}\right)^{1 / 2} p^{-\eta},
$$

for any $\eta<1 / 8$. The implicit constant depends only on $\eta$, on the implicit constants in (3.1) and on the conductor of $\mathcal{F}$.

This is Theorem 1.15 in [8], which depends essentially on methods of [7], to which we refer for more details and definitions concerning trace functions. For the purpose of this paper, it is sufficient to know that for any $k \geqslant 2$, any prime $p$ and $h \in \mathbb{F}_{p}^{\times}$, the functions given by

$$
K(a)=(-1)^{k-1} \mathrm{Kl}_{k}(a h ; p), \quad \text { for } a \in \mathbb{F}_{p}^{\times}, \quad K(0)=(-1)^{k} p^{-(k-1) / 2}
$$

are trace functions associated to geometrically irreducible sheaves $\mathcal{F}_{k, h}$ of rank $k$ with conductor bounded by a constant $C_{k}$ depending only on $k$, which is proved in [7, Prop. 10.3]. In fact, only the case $k=3$ will be used.

Another general result is the following estimate for general "type III" sums, which follows from our results in [8]. In the context of the function $d_{3}$, the corresponding trick of grouping variables appears in the work of HeathBrown (see [10, p. 42-43], where previous occurrences in the work of Yüh are mentioned).

THEOREM 3.2. Let $p$ be a prime, and let $K$ be the trace function of an $\ell$-adic middle-extension sheaf $\mathcal{F}$, pointwise of weight 0 , on the affine line over $\mathbb{F}_{p}$. Assume that $\mathcal{F}$ is geometrically irreducible and is not geometrically isomorphic to a tensor product of an Artin-Schreier sheaf associated to an additive character modulo $p$ and a multiplicative Kummer sheaf. 
For any complex coefficients $(\alpha(n))_{|n| \leqslant N_{1}},(\beta(n))_{|n| \leqslant N_{2}},(\gamma(n))_{|n| \leqslant N_{3}}$ with modulus less than 1 and any $\varepsilon>0$, we have

$$
\begin{aligned}
& \sum_{\substack{1 \leqslant\left|n_{i}\right| \leqslant N_{i} \\
p \nmid n_{3}}} \sum_{\substack{n_{1}\\
}} \alpha\left(n_{1}\right) \beta\left(n_{2}\right) \gamma\left(n_{3}\right) K\left(n_{1} n_{2} n_{3}\right) \\
& \ll(\log p)^{1 / 2}\left(N_{1} N_{2} N_{3}\right)^{1 / 2+\varepsilon}\left(\frac{N_{1} N_{2} N_{3}}{\sqrt{p}}+N_{1} N_{2}+N_{3} \sqrt{p}\right)^{1 / 2},
\end{aligned}
$$

where the implied constant depends only on $\varepsilon>0$ and polynomially on $\operatorname{cond}(\mathcal{F})$.

Proof. After elementary dyadic subdivisions (and summing over the separate signs), we see that it is enough to apply [8, Th. 1.16 (1)] with the choices

$$
\begin{array}{cl}
M=N_{3}, & N=N_{1} N_{2}, \\
\alpha_{m}=\gamma(m), & \beta_{n}=(\alpha \star \beta)(n)
\end{array}
$$

where $\star$ is the Dirichlet convolution. The bound we derive from $[\mathbf{8}]$ is

$$
\begin{aligned}
& \left(\sum_{m}|\gamma(m)|^{2}\right)^{1 / 2}\left(\sum_{n}|(\alpha \star \beta)(n)|^{2}\right)^{1 / 2}\left(N_{1} N_{2} N_{3}\right)^{1 / 2} \\
& \quad \times\left(\frac{1}{p^{1 / 4}}+\frac{1}{\sqrt{N_{3}}}+\frac{p^{1 / 4}(\log p)^{1 / 2}}{\sqrt{N_{1} N_{2}}}\right),
\end{aligned}
$$

and one checks easily that this implies the statement above.

Again we will only need to know that we can apply this to the functions $K$ above.

§4. Preliminary reductions. In this section, we will set up the proof of Theorem 1.1, in a way very similar to the preliminaries in [9] and [10]. The notational conventions that we introduce here will be valid throughout the remainder of the paper.

In $\S 4$ and in $\S 5$ the letter $q$ is reserved to denote a prime number, $x \geqslant 1$ is a real number, and we denote $\mathcal{L}=\log 2 x$ for simplicity. We define

$$
S(x ; q, a):=\sum_{\substack{n \equiv a \bmod q \\ n \leqslant x}} d_{3}(n)=\sum_{\substack{m_{1} m_{2} m_{3} \equiv a \bmod q \\ m_{1} m_{2} m_{3} \leqslant x}} 1,
$$

where $a$ is some integer coprime with $q$, and

$$
S^{*}(x ; q):=\sum_{\substack{n \leqslant x \\(n, q)=1}} d_{3}(n), \quad S(x)=\sum_{n \leqslant x} d_{3}(n) .
$$

If $q<x^{1 / 100}$, we have (1.1) trivially. Hence we can assume that

$$
x^{1 / 100} \leqslant q \leqslant x^{99 / 100}
$$


Since $q$ is prime, the assumption (4.1) implies

$$
S^{*}(x ; q)=S(x)+O_{\epsilon}\left(x^{99 / 100+\epsilon}\right),
$$

for every $\epsilon>0$. Moreover, $S(x)$ is of size $\frac{1}{2} x \mathcal{L}^{2}$, and hence Theorem 1.1 will follow if we prove that, for any $\theta<1 / 2+1 / 46$, we have

$$
S(x ; q, a)=\frac{1}{q} S(x)+O\left(\frac{x}{q \mathcal{L}^{A}}\right),
$$

for any $A>0$, uniformly for $a$ not divisible by $q$ such that $x^{1 / 100} \leqslant q \leqslant x^{\theta}$, the implied constant depending on $\theta$ and $A$.

We will need to make the three variables $m_{1}, m_{2}$ and $m_{3}$ independent and smooth. For this purpose, we use a smooth partition of unity, which is given by the following lemma (see [5, Lemme 2] for instance).

LEMMA 4.1. For every $\Delta>1$, there exists a sequence $\left(b_{\ell, \Delta}\right)_{\ell \geqslant 0}$ of smooth functions with support included in $\left[\Delta^{\ell-1}, \Delta^{\ell+1}\right]$, such that

$$
\sum_{\ell=0}^{\infty} b_{\ell, \Delta}(\xi)=1 \quad \text { for all } \xi \geqslant 1
$$

and

$$
b_{\ell, \Delta}^{(v)}(\xi) \ll_{v} \xi^{-v} \Delta^{v}(\Delta-1)^{-v} \text { for all } \xi \geqslant 1 \text { and } v \geqslant 0 .
$$

We take $\Delta$ slightly larger than 1 , namely

$$
\Delta=1+\mathcal{L}^{-B}
$$

for some parameter $B \geqslant 1$.

From now on, we denote by $M_{i}, 1 \leqslant i \leqslant 3$, some parameters of the form

$$
M_{i}=\Delta^{\ell}=\left(1+\mathcal{L}^{-B}\right)^{\ell},
$$

where $\ell \geqslant 0$ is an integer. For such a variable $M_{i}=\Delta^{\ell}$, we define

$$
V_{i}(t)=b_{\ell, \Delta}(t),
$$

where $b_{\ell, \Delta}$ are the functions given by Lemma 4.1. Thus, the derivatives of $V_{i}$ satisfy

$$
V_{i}^{(v)}(t) \ll v t^{-v} \mathcal{L}^{B v}
$$

The bound (4.7) implies the classical fact that $\widehat{V}_{i}(\xi)$ decays quickly, namely

$$
\widehat{V}_{i}(\xi) \ll_{v} M_{i}\left(\frac{\mathcal{L}^{B}}{|\xi| M_{i}}\right)^{v}
$$

for all integers $v \geqslant 0$ and $\xi \neq 0$.

For $\boldsymbol{M}=\left(M_{1}, M_{2}, M_{3}\right)$, we can now consider the smooth sums

$$
S(\boldsymbol{M} ; q, a)=\sum_{m_{1} m_{2}} \sum_{m_{3} \equiv a} \sum_{\bmod q} V_{1}\left(m_{1}\right) V_{2}\left(m_{2}\right) V_{3}\left(m_{3}\right),
$$


and

$$
S(\boldsymbol{M})=\sum_{m_{1}, m_{2}, m_{3}} \sum_{1} V_{1}\left(m_{1}\right) V_{2}\left(m_{2}\right) V_{3}\left(m_{3}\right) .
$$

Our preparation for Theorem 1.1 is given by the following lemma.

LEMMA 4.2. For any $A>0$, we can select $B \geqslant 1$ such that

$$
S(x, q ; a)-\frac{1}{q} S(x)=\sum_{\boldsymbol{M}}\left(S(\boldsymbol{M} ; q, a)-\frac{1}{q} S(\boldsymbol{M})\right)+O_{\theta}\left(q^{-1} x \mathcal{L}^{-A}\right),
$$

where $\boldsymbol{M}=\left(M_{1}, M_{2}, M_{3}\right)$ runs over triples of $M_{i}$ as above such that

$$
x \mathcal{L}^{-B} \leqslant M_{1} M_{2} M_{3} \leqslant x .
$$

Proof. Using the partition of unity above, we have

$$
\begin{aligned}
S(x ; q, a) & \left.=\sum_{\boldsymbol{M}=\left(M_{1}, M_{2}, M_{3}\right)} \sum_{\substack{ \\
x \leqslant n \leqslant x \Delta^{3}}} d_{3}(n)\right) \\
& =\sum_{\boldsymbol{M}=\left(M_{1}, M_{2}, M_{3}\right)} \sum_{\substack{x \equiv a \bmod q \\
n}} S(\boldsymbol{M} ; q, a)+O\left(x q^{-1} \mathcal{L}^{2-B}\right),
\end{aligned}
$$

where the sum ranges over all the triples $\boldsymbol{M}=\left(M_{1}, M_{2}, M_{3}\right)$ of the form above such that $M_{1} M_{2} M_{3} \leqslant x$, and the bound on the error term is based on a classical estimate for the sum of the divisor function in arithmetic progressions, restricted to an interval (see [14, Theorem 2] for instance).

Similarly, the contribution to this sum of the triples $\left(M_{1}, M_{2}, M_{3}\right)$ satisfying $M_{1} M_{2} M_{3} \leqslant x \mathcal{L}^{-B}$ satisfies

$$
\sum_{M_{1} M_{2} M_{3} \leqslant x \mathcal{L}^{-B}} S(\boldsymbol{M} ; q, a) \leqslant \sum_{\substack{1 \leqslant n \leqslant 2 x \mathcal{L}^{-B} \\ n \equiv a \bmod q}} d_{3}(n) \ll x q^{-1} \mathcal{L}^{2-B} .
$$

Thus by selecting $B=B(A)$ large enough in (4.12) and (4.13), we get

$$
S(x ; q, a)=\sum_{\left(M_{1}, M_{2}, M_{3}\right)} \sum_{M_{1}} S(\boldsymbol{M} ; q, a)+O\left(x q^{-1} \mathcal{L}^{-A}\right),
$$

where the sum is over the triples $\left(M_{1}, M_{2}, M_{3}\right)$ such that (4.11) holds. A similar result holds for the sum $S(x)$, and gives the result.

Due to the symmetry of the problem, it is natural to introduce the following condition

$$
M_{3} \geqslant M_{2} \geqslant M_{1}
$$

Since the number of triples $\boldsymbol{M}$ satisfying (4.11) with $M_{i}$ of the form (4.5) is $\ll \mathcal{L}^{3 B+3}$, Lemma 4.2 shows that (4.3) (and hence Theorem 1.1) will follow if we can show that for any $\theta<1 / 2+1 / 46$ and $A>0$, we have

$$
S(\boldsymbol{M} ; q, a)=\frac{1}{q} S(\boldsymbol{M})+O_{\theta}\left(q^{-1} x \mathcal{L}^{-A}\right),
$$


uniformly for all triples $\boldsymbol{M}=\left(M_{1}, M_{2}, M_{3}\right)$ satisfying (4.11), (4.14) and (4.5) and for all integers $a$ coprime with $q$ satisfying $x^{1 / 100} \leqslant q \leqslant x^{\theta}$. The proof of this is the object of the next section.

§5. Conclusion of the proof of Theorem 1.1. The first two subsections below establish estimates for $S(\boldsymbol{M} ; q, a)$ which are non-trivial in two different ranges, depending on the sizes of $M_{1}, M_{2}, M_{3}$. In the last subsection, we combine them to derive (4.15).

In order to present the two cases cleanly, we introduce the parameters $\kappa$ and $\mu_{i}$ defined by

$$
q=x^{\kappa} \quad \text { and } \quad M_{i}=x^{\mu_{i}} \quad \text { for } 1 \leqslant i \leqslant 3,
$$

so that $\kappa$ and $\mu_{i}$ satisfy

$$
1 / 100 \leqslant \kappa \leqslant 99 / 100
$$

and

$$
1-B \frac{\log \mathcal{L}}{\mathcal{L}} \leqslant \mu_{1}+\mu_{2}+\mu_{3} \leqslant 1, \mu_{3} \geqslant \mu_{2} \geqslant \mu_{1} \geqslant 0,
$$

as a consequence of (4.1), (4.11) and (4.14). We also remind the reader that $q$ denotes a prime number.

5.1. Applying the combined summation formula. We apply the combined summation formula of Corollary 2.3 to $S(\boldsymbol{M} ; q, a)$, which is of the form treated there with $K(n)$ the characteristic function of the residue class $a \bmod q$. We then have

$$
\widehat{K}(0)=\frac{1}{\sqrt{q}},
$$

and, for $(q, n)=1$,

$$
\widetilde{K}(x, n)=\frac{\mathrm{Kl}_{3}(\text { anx } ; q)}{\sqrt{q}},
$$

by Lemma 2.4. We therefore get the equality

$$
S(\boldsymbol{M} ; q, a)=\mathrm{A}+\mathrm{B}+\mathrm{C}+\mathrm{D},
$$

as in Corollary 2.3, and we proceed to handle these four terms.

First of all, we have

$$
\mathrm{A}=\frac{1}{q} \sum_{\substack{\left(m_{1} m_{2}, q\right)=1 \\ m_{3} \geqslant 1}} \sum_{1} V_{1}\left(m_{1}\right) V_{2}\left(m_{2}\right) V_{3}\left(m_{3}\right)=\frac{1}{q} S(\boldsymbol{M})+O\left(\frac{x}{q^{2}}\right),
$$

which represents the desired main term. We will now find conditions which ensure that $B, C$ and $D$ are small. We will use the inequality

$$
\widehat{V}_{i}(t) \ll M_{i},
$$

several times (see (4.8)). 
First, we have

$$
\mathrm{B}=-\frac{1}{q^{2}} \sum_{\substack{m_{1}, m_{2}, n_{3} \\\left(n_{3}, q\right)=1}} \sum V_{1}\left(m_{1}\right) V_{2}\left(m_{2}\right) \widehat{V}_{3}\left(\frac{n_{3}}{q}\right),
$$

and by applying Lemma 2.1 twice, we get

$$
\begin{aligned}
\sum_{\left(n_{3}, q\right)=1} \hat{V}_{3}\left(\frac{n_{3}}{q}\right) & =\sum_{n_{3}} \hat{V}_{3}\left(\frac{n_{3}}{q}\right)-\sum_{q \mid n_{3}} \widehat{V}_{3}\left(\frac{n_{3}}{q}\right) \\
& =q \sum_{t \equiv 0 \bmod q} V_{3}(t)-\sum_{t} V_{3}(t) \ll M_{3},
\end{aligned}
$$

by the properties of the function $V_{3}$. Inserting this bound into (5.6) and combining with (4.11), we deduce

$$
\mathrm{B} \ll q^{-2} x
$$

Similarly, using the definition

$$
\begin{aligned}
\mathrm{C}= & \frac{1}{q^{5 / 2}}\left\{\widehat{V}_{1}(0) \sum_{n_{2}} \widehat{V}_{2}\left(\frac{n_{2}}{q}\right)+\widehat{V}_{2}(0) \sum_{n_{1}} \widehat{V}_{1}\left(\frac{n_{1}}{q}\right)-\widehat{V}_{1}(0) \widehat{V}_{2}(0)\right\} \\
& \times \sum_{\left(n_{3}, q\right)=1} \widehat{V}_{3}\left(\frac{n_{3}}{q}\right),
\end{aligned}
$$

a computation similar to (5.7) leads to

$$
\mathrm{C} \ll q^{-5 / 2} x
$$

We must now only deal with D. By Lemma 2.4 , we can write

$$
\mathrm{D}=\frac{1}{q^{2}} \sum \sum_{\substack{n_{1} n_{2} \neq 0 \\\left(n_{3}, q\right)=1}} \sum \widehat{V}_{1}\left(\frac{n_{1}}{q}\right) \widehat{V}_{2}\left(\frac{n_{2}}{q}\right) \widehat{V}_{3}\left(\frac{n_{3}}{q}\right) \mathrm{Kl}_{3}\left(a n_{1} n_{2} n_{3} ; q\right) \text {. }
$$

For fixed $n_{3}$, the sum over $n_{1}$ and $n_{2}$ can be handled using Theorem 3.1, according to the remark after (3.2), except that the Fourier transforms of the functions $V_{i}$ are not compactly supported. To handle this minor difficulty, we use a partition of unity again. Precisely, we apply Lemma 4.1 with parameter $\Delta=2$, deriving a decomposition

$$
\mathrm{D}=\frac{1}{q^{2}} \sum_{N} \mathcal{D}(N)
$$

where $N$ runs over triples $N=\left(N_{1}, N_{2}, N_{3}\right), N_{i}$ are integers of the form $2^{\ell}$ for some $\ell \geqslant 0$, and

$$
\begin{aligned}
\mathcal{D}(N)= & \sum \sum_{\substack{n_{1} n_{2} \neq 0 \\
\left(n_{3}, q\right)=1}} \sum\left(\widehat{V}_{1}\left(\frac{n_{1}}{q}\right) W_{1}\left(n_{1}\right)\right)\left(\widehat{V}_{2}\left(\frac{n_{2}}{q}\right) W_{2}\left(n_{2}\right)\right) \\
& \times\left(\widehat{V}_{3}\left(\frac{n_{3}}{q}\right) W_{3}\left(n_{3}\right)\right) \mathrm{Kl}_{3}\left(a n_{1} n_{2} n_{3} ; q\right)
\end{aligned}
$$

where $W_{j}(t)=b_{\ell, 2}(t)$, a smooth function supported in[ $\left[N_{j} / 2, N_{j}\right]$. 
The inequality (4.8) implies that the coefficients $n_{i} \mapsto \widehat{V}_{i}\left(n_{i} / q\right)$ decay quickly as soon as

$$
n_{i}>\tilde{N}_{i}=q M_{i}^{-1} x^{\eta}
$$

where $\eta>0$ is arbitrarily small. Thus we get

$$
\mathrm{D}=\frac{1}{q^{2}} \sum_{\substack{N \\ N_{i} \leqslant \tilde{N}_{i}}} \mathcal{D}(N)+O_{\eta}\left(x^{-1}\right) .
$$

The sum over $N$ contains $\ll \mathcal{L}^{3}$ terms. By this remark and by the relations (4.1), (5.3), (5.4), (5.8)-(5.10), we see that it is enough (in order to prove (4.15)) to show that

$$
\mathcal{D}(N) \ll_{\epsilon, A} q x \mathcal{L}^{-A},
$$

for all $\epsilon>0$, all $A>0$, all $\boldsymbol{M}$ satisfying (5.1) and (5.2), all $N_{i} \leqslant \tilde{N}_{i}$ and all $q=x^{\kappa}$ where $1 / 100 \leqslant \kappa \leqslant 12 / 23-\epsilon$.

We apply Theorem 3.1 to the sum over $\left(n_{1}, n_{2}\right)$ in $\mathcal{D}(\boldsymbol{N})$. This means that, in that result, we take parameters

$$
\begin{gathered}
\left(M_{1}, M_{2}\right)=\left(N_{1}, N_{2}\right), \quad K(n)=\mathrm{Kl}_{3}\left(a n_{3} n ; q\right) \\
V(x)=M_{1}^{-1} \widehat{V}_{1}\left(x N_{1} / q\right) W_{1}\left(x N_{1}\right), \quad W(x)=M_{2}^{-1} \widehat{V}_{2}\left(x N_{2} / q\right) W_{2}\left(x N_{2}\right),
\end{gathered}
$$

which ensure that (3.1) holds with $Q=x^{2 \eta}$, and we must multiply the resulting bound by $M_{1} M_{2}$.

Since, in addition, we have already observed that the conductor of $n \mapsto$ $\mathrm{Kl}_{3}\left(a n_{3} n ; q\right)$ is bounded by an absolute constant, we obtain the upper bound

$$
\mathcal{D}(\boldsymbol{N}) \ll_{\eta} M_{1} M_{2} M_{3} N_{1} N_{2} N_{3}\left(1+\frac{q}{N_{1} N_{2}}\right)^{1 / 2} q^{-1 / 8} x^{3 \eta}
$$

after applying Theorem 3.1 and summing trivially over $n_{3}$.

This bound is worst when $N_{i}=\tilde{N}_{i}=q M_{i}^{-1} x^{\eta}$. Hence, using (5.2), this implies

$$
\mathcal{D}(\boldsymbol{N}) \ll_{\eta}\left(1+\frac{x}{q M_{3}}\right)^{1 / 2} q^{23 / 8} x^{6 \eta} .
$$

It follows easily that (5.11) is satisfied as soon as

$$
\kappa \leqslant \frac{8}{15}-4 \eta \text { and } \mu_{3} \geqslant \frac{11}{4} \kappa-1+14 \eta .
$$

This is our first estimate.

5.2. Grouping variables. In the totally symmetric situation where

$$
\mu_{1}=\mu_{2}=\mu_{3}=1 / 3
$$

the inequalities (5.12) are very restrictive and do not allow us to extend the value of the exponent of distribution beyond $1 / 2$. Instead, we use Theorem 3.2 (which builds on the construction of a long variable by grouping two short ones). 
We obtain (see again (5.5))

$\mathcal{D}(N) \ll_{\eta}\left(M_{1} M_{2} M_{3}\right)\left(N_{1} N_{2} N_{3}\right)^{1 / 2}\left(q^{-1 / 2} N_{1} N_{2} N_{3}+N_{1} N_{2}+q^{1 / 2} N_{3}\right)^{1 / 2} x^{\eta}$.

The right-hand side is a non-decreasing function of the parameters $N_{i} \leqslant \tilde{N}_{i}$, and it leads to

$$
\begin{aligned}
\mathcal{D}(\boldsymbol{N}) & \ll_{\eta} x \cdot\left(q^{3} / x\right)^{1 / 2}\left(q^{-1 / 2}\left(q^{3} / x\right)+M_{3}\left(q^{2} / x\right)+q^{3 / 2} M_{3}^{-1}\right)^{1 / 2} x^{5 \eta} \\
& \ll\left(q^{11 / 4}+q^{5 / 2} M_{3}^{1 / 2}+q^{9 / 4} x^{1 / 2} M_{3}^{-1 / 2}\right) x^{5 \eta} .
\end{aligned}
$$

This implies that (5.11) is also satisfied when we have

$$
\kappa \leqslant \frac{4}{7}-\eta, \quad \frac{5}{2} \kappa-1+12 \eta \leqslant \mu_{3} \leqslant 2-3 \kappa-12 \eta .
$$

5.3. End of the proof of Theorem 1.1. For the final step, we combine the results of the last two subsections. Choosing $\eta=\epsilon / 10$ for $\epsilon>0$ very small, we see that whenever

$$
\kappa \leqslant 1 / 2+1 / 46-\epsilon
$$

we have

$$
\frac{11}{4} \kappa-1+14 \eta \leqslant 2-3 \kappa-12 \eta .
$$

Looking at the conditions in (5.12) and (5.13), we see that the bound (5.11) holds provided that

$$
\mu_{3} \geqslant \frac{5}{2} \kappa-1+2 \epsilon
$$

But by (5.2), we have

$$
\mu_{3} \geqslant \frac{1}{3}-\frac{B \log \mathcal{L}}{3 \mathcal{L}} \geqslant \frac{5}{2} \kappa-1+2 \epsilon
$$

for $x$ large enough. This completes the proof of Theorem 1.1.

Remark 5.1. The exponent $1 / 2+1 / 46$ is best possible using only the conditions (5.12) and (5.13) that arise from Theorems 3.1 and 3.2. Indeed, neither applies to the triple $\left(\mu_{1}, \mu_{2}, \mu_{3}\right)=(13 / 46,13 / 46,10 / 23)$.

§6. Proof of Theorem 1.2. We will now prove Theorem 1.2, concerning $d_{3}$ on integers congruent to a fixed integer $a \neq 0$, modulo $q$, on average over $q \leqslant Q$. We start by elementary reductions.

In addition to the sums $S(\boldsymbol{M} ; q, a)$ and $S(\boldsymbol{M})$ which are defined in (4.9) and (4.10), we also consider

$$
S^{*}(\boldsymbol{M}, q)=\sum_{\left(m_{1} m_{2} m_{3}, q\right)=1} \sum_{1} V_{1}\left(m_{1}\right) V_{2}\left(m_{2}\right) V_{3}\left(m_{3}\right)
$$

Then, for a prime $q$ satisfying (4.1) and a triple $\boldsymbol{M}$ satisfying (4.11), we have

$$
\frac{1}{q} S(\boldsymbol{M})=\frac{1}{\varphi(q)} S^{*}(\boldsymbol{M} ; q)+O_{\epsilon}\left(\frac{x^{99 / 100+\epsilon}}{q}\right)
$$


(compare with (4.2)). Using the reductions of $\$ 4$ (in particular Lemma 4.2) and Theorem 1.1, we see that Theorem 1.2 follows from the (equivalent) estimates

$$
\begin{aligned}
& \sum_{\substack{q \sim Q \\
q \text { prime, } q \nmid a}}\left|S(\boldsymbol{M} ; q, a)-\frac{1}{q} S(\boldsymbol{M})\right| \ll x \mathcal{L}^{-A}, \\
& \sum_{\substack{q \sim Q \\
q \text { prime }, q \nmid a}}\left|S(\boldsymbol{M} ; q, a)-\frac{1}{\varphi(q)} S^{*}(\boldsymbol{M}, q)\right| \ll x \mathcal{L}^{-A},
\end{aligned}
$$

which are valid for every $A>0$ and $B>0$, every triple $\boldsymbol{M}$ (subject to (4.11), (4.14) and (4.5)), and all $Q$ in a range

$$
x^{12 / 23-\alpha} \leqslant Q \leqslant x^{9 / 17-\alpha}
$$

for some $\alpha>0$, where the implied constant may depend only on $(\alpha, A, B)$ (it would even be enough to do it for each $A$ with $B$ depending on $A$ ).

We will establish these bounds in two steps: another individual estimate for each $q$, which follows from the previous sections, and a final bound on average for which we use Kloostermania [3].

6.1. Reduction to Kloostermania. The first estimate is given by the following proposition.

Proposition 6.1 (Individual bound). With notation as above, for $\boldsymbol{M}=$ $\left(M_{1}, M_{2}, M_{3}\right)$ satisfying (4.11) and (4.14), for every $B>0$, every $\eta>0$ and $\alpha>0$ and every prime $q$ such that $x^{12 / 23-\alpha} \leqslant q \leqslant x^{9 / 17-\alpha}$ and

$$
q^{5 / 2} x^{-1+\eta} \leqslant M_{3} \leqslant q^{-3} x^{2-\eta} \quad \text { or } \quad M_{3} \geqslant q^{11 / 4} x^{-1+\eta},
$$

we have

$$
S(\boldsymbol{M} ; q, a)=\frac{1}{q} S(\boldsymbol{M})+O\left(q^{-1} x^{1-\eta_{1}}\right),
$$

for some $\eta_{1}>0$ depending only on $\eta$, where the implied constant depends only on $(\eta, \alpha, B)$.

Proof. This is an immediate consequence of (5.12) and (5.13).

Our second estimate is on average over $q$; we will obtain stronger bounds, and we do not require $q$ to be restricted to primes, but on the other hand, we now need to fix $a$.

Proposition 6.2 (Average bound). Let $a \neq 0$ be a fixed integer. For every $\eta>0$ there exists $\eta_{1}>0$, depending only on $\eta$, such that for every $\boldsymbol{M}$ as above satisfying

$$
x^{11 / 23} \geqslant M_{3} \geqslant M_{2} \geqslant M_{1}
$$

and for every $Q$ such that

$$
Q \leqslant x^{-\eta} \min \left\{x M_{3}^{-1}, x^{-1 / 2} M_{3}^{5 / 2}, x^{1 / 4} M_{3}^{3 / 4}\right\}
$$


we have

$$
\sum_{\substack{q \sim Q \\(q, a)=1}}\left|S(\boldsymbol{M} ; q, a)-\frac{1}{\varphi(q)} S^{*}(\boldsymbol{M}, q)\right| \ll x^{1-\eta_{1}}
$$

where the implied constant depends only on $(a, B, \eta)$.

Before giving the proof, we combine these two results.

Proof of (6.1) and (6.2). Summing (6.3) over all primes $q \sim Q$, we obtain (6.1) when

$$
x^{12 / 23-\alpha} \leqslant Q \leqslant x^{9 / 17-\alpha}
$$

for some fixed $\alpha>0$ and

$$
Q^{5 / 2} x^{-1+\eta} \leqslant M_{3} \leqslant Q^{-3} x^{2-\eta} \quad \text { or } \quad M_{3} \geqslant Q^{11 / 4} x^{-1+\eta},
$$

for some fixed $\eta>0$.

Fixing $\alpha>0$ and $\eta=\alpha$, assuming that (6.7) holds, it is therefore enough to show that (6.2) holds when

$$
Q^{-3} x^{2-\alpha} \leqslant M_{3} \leqslant Q^{11 / 4} x^{-1+\alpha} .
$$

We claim that under these assumptions, if $\alpha$ is small enough, Proposition 6.2 can be applied for the value of the parameter $\eta=\alpha / 2$. We then derive (6.6) by Proposition 6.2 for some $\eta_{1}>0$, and this is stronger than (6.2).

To check the claim, note first that the condition (6.4) is clear from the assumptions (6.7) and (6.8) if $\alpha$ is small enough. Moreover:

- $\quad$ since $M_{3} \leqslant Q^{11 / 4} x^{-1+\alpha}$ and $Q \leqslant x^{9 / 17}$, we have $M_{3} Q \leqslant x^{1-1 / 68+\alpha} \leqslant$ $x^{1-\eta}$ for $\alpha$ small enough;

- $\quad$ since $M_{3} \geqslant Q^{-3} x^{2-\alpha}$ and $Q \leqslant x^{9 / 17-\alpha}$, we have $Q \leqslant x^{-1 / 2-\eta} M_{3}^{5 / 2}$, and also $Q \leqslant x^{1 / 4-\eta} M_{3}^{3 / 4}$.

This means that (6.5) is also valid, as claimed.

\$7. Proof of Proposition 6.2. We denote by $\Sigma(Q, \boldsymbol{M}, a)$ the left-hand side of (6.6). Denoting further by $c_{q}$ the sign of the difference

$$
S(\boldsymbol{M} ; q, a)-\frac{S^{*}(\boldsymbol{M})}{\varphi(q)}
$$

when $(q, a)=1$, and putting $c_{q}=0$ when $a$ is not coprime to $q$, we can write

$$
\Sigma(Q, \boldsymbol{M}, a)=\Sigma_{0}(Q, \boldsymbol{M}, a)-\Sigma_{1}(Q, \boldsymbol{M}, a),
$$

where

$$
\Sigma_{0}(Q, \boldsymbol{M}, a)=\sum_{q \sim Q} c_{q} S(\boldsymbol{M} ; q, a), \quad \Sigma_{1}(Q, \boldsymbol{M}, a)=\sum_{q \sim Q} \frac{c_{q}}{\varphi(q)} S^{*}(\boldsymbol{M} ; q) .
$$


7.1. Evaluation of $\Sigma_{1}(Q, \boldsymbol{M}, a)$. In this section we obtain an asymptotic formula for $\Sigma_{1}$.

LEMMA 7.1. With notation and assumptions as above, for any complex numbers $\sigma_{q}$ with $\left|\sigma_{q}\right| \leqslant 1$, we have

$$
\begin{aligned}
\sum_{q \sim Q} \frac{\sigma_{q}}{\varphi(q)} S^{*}(M ; q)= & \widehat{V}_{1}(0) \widehat{V}_{2}(0) \widehat{V}_{3}(0) \sum_{q \sim Q} \frac{\sigma_{q}}{\varphi(q)} \cdot\left(\frac{\varphi(q)}{q}\right)^{3} \\
& +O\left(M_{2} M_{3} d^{3}(q) \mathcal{L}^{6 B}\right)
\end{aligned}
$$

where $V_{i}$ are the functions appearing in the definition of $S(\boldsymbol{M} ; q, a)$.

In view of the definition (and the fact that $M_{1} \leqslant M_{2} \leqslant M_{3}$ ), this follows from the following lemma, which we state in slightly greater generality for later use.

LEMMA 7.2. Let $V=V_{i}$ for some $1 \leqslant i \leqslant 3$ as in (4.6). Then for any integer $u \geqslant 1$ and any integer $q \geqslant 1$, we have

$$
\sum_{\left(m_{i}, q\right)=1} V_{i}\left(u m_{i}\right)=\frac{\varphi(q)}{q u} \widehat{V}_{i}(0)+O\left(d(q) \mathcal{L}^{2 B}\right) .
$$

Proof. If we write $W(t)=V_{i}(t u)$ for $t \in \mathbb{R}$, we see that $W(t)=0$ for $|t| \geqslant 2 M_{i} / u$ and that $\widehat{W}(t)=(1 / u) \widehat{V}_{i}(t / u)$. We then apply the Möbius inversion formula, the Poisson formula (Lemma 2.1) and (4.8) (with $v=2$ ) to get

$$
\begin{aligned}
\sum_{\left(m_{i}, q\right)=1} V_{i}\left(u m_{i}\right) & =\sum_{\substack{d \mid q \\
d \leqslant 2 M_{i} / u}} \mu(d) \sum_{d \mid m_{i}} W\left(m_{i}\right)=\sum_{\substack{d \mid q \\
d \leqslant 2 M_{i} / u}} \frac{\mu(d)}{d u} \sum_{n} \widehat{V}_{i}\left(\frac{n}{d u}\right) \\
& =\sum_{\substack{d \mid q \\
d \leqslant 2 M_{i} / u}} \frac{\mu(d)}{d u}\left\{\widehat{V}_{i}(0)+O\left(M_{i} \sum_{|n| \geqslant 1}\left(d u n^{-1} M_{i}^{-1} \mathcal{L}^{B}\right)^{2}\right)\right\},
\end{aligned}
$$

and the lemma follows after summing over $n$ and $d$.

7.2. Application of Kloostermania. The treatment of $\Sigma_{0}(Q, \boldsymbol{M}, a)$ is more intricate. Obviously the problem of proving (6.6) essentially deals with the average distribution of the convolution of two (or three) arithmetic functions in arithmetic progressions. Thirty years ago, this problem was considered in a series of papers by Bombieri, Fouvry, Friedlander and Iwaniec (see in particular $[6,4,1,2]$ ) with the purpose of improving the exponent $1 / 2$ in the classical Bombieri-Vinogradov theorem concerning the distribution of primes in arithmetic progressions (see [11, Theorem 17.1] for instance).

These investigations resulted in several variants of the Bombieri-Vinogradov theorem, with well-factorable coefficients in the averaging and with exponents of distribution greater than $1 / 2$, culminating with the exponent 4/7 [1, Theorem 10]. The crucial ingredient was the use of the so-called Kloostermania, i.e. estimates for sums of Kloosterman sums arising from the Kuznetsov formula 
and from the spectral theory of modular forms on congruence subgroups, which was developed in the seminal work of Deshouillers and Iwaniec [3].

Among the currently known results, the following estimate is well suited to our problem.

PROPOSITION 7.3 (Bombieri-Friedlander-Iwaniec). Let $a \neq 0$ be an integer. Let $f$ be a $C^{1}$ complex-valued function defined on $\mathbb{R}$ with $|f| \ll 1$. For every $\eta>0$ there exists $\eta_{1}>0$, depending only on $\eta$, such that for all sequences $\left(\gamma_{q}\right)$, $\left(\delta_{r}\right)$ and $\left(\beta_{n}\right)$ of complex numbers of modulus at most 1 and for all parameters

$$
x, M, N, Q, R \geqslant 1
$$

such that $Q R<x, M N=x$ and

$$
x^{1-\eta}>M>x^{\eta} \max \left\{Q, x^{-1} Q R^{4}, Q^{1 / 2} R, x^{-2} Q^{3} R^{4}\right\},
$$

we have

$$
\begin{aligned}
& \sum_{\substack{q \sim Q \\
(q r, a)=1}} \sum_{r \sim R} \gamma_{q} \delta_{r}\left(\sum_{\substack{m \sim M \\
m n \equiv a \bmod q r}} \sum_{\substack{n \sim N \\
m n}} \beta_{n} f(m)-\frac{1}{\varphi(q r)} \sum_{\substack{m \sim M \\
(m n, q r)=1}} \sum_{n \sim N} \beta_{n} f(m)\right) \\
& \quad=O\left(x^{1-\eta_{1}}\left(1+\sup _{|t| \sim M}\left|f^{\prime}(t)\right|\right)\right),
\end{aligned}
$$

where the implied constant depends only on $\eta$, a and $\sup _{t}|f(t)|$.

Proof. This follows very easily from [1, Theorem 5], which is the case $f=1$, after summation by parts; one should just notice that the argument in [1, p. 235, 236] applies equally well when $\alpha_{m}=1$ for $m$ in a sub-interval $I \subset[M, 2 M]$ and $\alpha_{m}=0$ for $m \sim M$ and $m \notin I$.

In order to apply this proposition we need to transform $\Sigma_{0}(Q, \boldsymbol{M}, a)$. For this purpose, we use a trick already present in [5, p. 75] (for instance), which consists in rewriting a congruence to a different modulus: the congruence

$$
m_{1} m_{2} m_{3} \equiv a \bmod q
$$

which appears in our sum $S(\boldsymbol{M} ; q, a)$ (see (4.9)) is reinterpreted as

$$
q r \equiv-a \bmod m_{1} m_{2} \text {. }
$$

A technical point is that we must preserve the coprimality condition $\left(m_{1} m_{2}\right.$, $a)=1$. To avoid complication, we begin with the case $a=1$, where this technical issue does not arise, and postpone a short discussion of the general case to $§ 7.3$.

For $a=1$, we therefore write

$$
\Sigma_{0}(Q, \boldsymbol{M}, 1)=\sum_{m_{1}} \sum_{m_{2}} V_{1}\left(m_{1}\right) V_{2}\left(m_{2}\right) \sum_{\substack{q \sim Q, r \\ q r \equiv-1 \bmod m_{1} m_{2}}} c_{q} V_{3}\left(\frac{q r+1}{m_{1} m_{2}}\right) .
$$

By (4.7) (with $v=1$ ) and (4.11), we have

$$
V_{3}\left(\frac{q r+1}{m_{1} m_{2}}\right)=V_{3}\left(\frac{q r}{m_{1} m_{2}}\right)+O\left(x^{-1} \mathcal{L}^{2 B}\right),
$$


and hence

$$
\begin{aligned}
\Sigma_{0}(Q, \boldsymbol{M}, 1)= & \sum_{m_{1}} \sum_{m_{2}} V_{1}\left(m_{1}\right) V_{2}\left(m_{2}\right) \sum_{\substack{q \sim Q, r \\
q r \equiv-1 \bmod m_{1} m_{2}}} c_{q} V_{3}\left(\frac{q r}{m_{1} m_{2}}\right) \\
& +O_{B}\left(\mathcal{L}^{2 B+2}\right) .
\end{aligned}
$$

This expression is close to the desired shape, but we must separate the variables $m_{1}, m_{2}, q$ and $r$ before we can apply Proposition 7.3. We use the Mellin transform for this purpose.

First, since $V_{3}$ is supported in $\left[M_{3}, 2 M_{3}\right]$, the variable $r$ satisfies

$$
R \ll r \ll R \quad \text { where } R=M_{1} M_{2} M_{3} Q^{-1} .
$$

We have

$$
V_{3}(\xi)=\frac{1}{2 \pi \mathrm{i}} \int_{(\sigma)} F_{3}(s) \xi^{-s} d s,
$$

for any fixed real number $\sigma$, where

$$
F_{3}(s)=\int_{0}^{\infty} V_{3}(\xi) \xi^{s-1} d \xi
$$

is the Mellin transform of $V_{3}$. This is an entire function of $s \in \mathbb{C}$ which satisfies

$$
F_{3}(\sigma+\mathrm{i} t) \ll_{k, \sigma}|t|^{-k} M_{3}^{\sigma} \mathcal{L}^{k B},
$$

for all $k \geqslant 1$, all $\sigma \in \mathbb{R}$ and $|t| \geqslant 1$ (as follows by repeated integrations by parts).

Let $v>0$ be a small parameter to be chosen later, and let

$$
T=x^{v} .
$$

Then, inserting (7.7) into (7.5) and applying (7.8) for $k$ large enough depending on $v$, we deduce that

$$
\begin{aligned}
\Sigma_{0}(Q, \boldsymbol{M}, 1)= & \frac{1}{2 \pi \mathrm{i}} \int_{-\mathrm{i} T}^{\mathrm{i} T} F_{3}(\mathrm{i} t) \sum_{m_{1}} \sum_{m_{2}}\left(V_{1}\left(m_{1}\right) m_{1}^{\mathrm{i} t}\right) \cdot\left(V_{2}\left(m_{2}\right) m_{2}^{\mathrm{i} t}\right) \\
& \times \sum_{\substack{q \sim Q, r \\
q r \equiv-1 \bmod m_{1} m_{2}}}\left(c_{q} q^{-\mathrm{i} t}\right) \cdot r^{-\mathrm{i} t} d t+O\left(\mathcal{L}^{2 B+2}\right),
\end{aligned}
$$

where the implied constant depends on $v$ and $B$.

For each $t$, we will apply Proposition 7.3 with

$$
\begin{gathered}
(Q, R, N, M) \leftrightarrow\left(M_{2}, M_{1}, Q, R\right), \\
\gamma_{q}=V_{2}(q) q^{\mathrm{i} t}, \quad \delta_{r}=V_{1}(r) r^{\mathrm{i} t}, \quad \beta_{n}=c_{n} n^{-\mathrm{i} t}, \\
m=r, \quad f(m)=F_{3}(\mathrm{i} t) m^{-\mathrm{i} t} .
\end{gathered}
$$


To do this, we must check that the conditions (7.2) are satisfied for these parameters. For a given $\eta>0$, using (7.6), these conditions translate to

$$
x^{1-\eta} \geqslant M_{1} M_{2} M_{3} Q^{-1} \geqslant x^{\eta} \max \left\{M_{2}, x^{-1} M_{2} M_{1}^{4}, M_{2}^{1 / 2} M_{1}, x^{-2} M_{2}^{3} M_{1}^{4}\right\} .
$$

By the assumption (4.11) and the inequality $Q>x^{12 / 23-\alpha}$, we see that these inequalities hold as soon as we have

$$
Q \leqslant x^{-2 \eta} \min \left\{x M_{2}^{-1}, x^{2} M_{1}^{-4} M_{2}^{-1}, x M_{1}^{-1} M_{2}^{-1 / 2}, x^{3} M_{1}^{-4} M_{2}^{-3}\right\} .
$$

From $M_{1} M_{2} M_{3} \leqslant x$ and $M_{1} \leqslant M_{2} \leqslant M_{3}$, we know that $M_{1} \leqslant\left(x / M_{3}\right)^{1 / 2}$, and from this we obtain

$$
\begin{gathered}
M_{2} \leqslant M_{3}, \\
M_{1}^{4} M_{2} \leqslant M_{1}^{3}\left(x / M_{3}\right) \leqslant\left(x / M_{3}\right)^{3 / 2}\left(x / M_{3}\right)=x^{5 / 2} M_{3}^{-5 / 2}, \\
M_{1} M_{2}^{1 / 2} \leqslant M_{1}^{1 / 2}\left(x / M_{3}\right)^{1 / 2} \leqslant\left(x / M_{3}\right)^{1 / 4}\left(x / M_{3}\right)^{1 / 2}=x^{3 / 4} M_{3}^{-3 / 4}, \\
M_{1}^{4} M_{2}^{3} \leqslant M_{1}\left(x / M_{3}\right)^{3} \leqslant\left(x / M_{3}\right)^{1 / 2}\left(x / M_{3}\right)^{3}=x^{7 / 2} M_{3}^{-7 / 2} .
\end{gathered}
$$

Hence (7.9) is satisfied as soon as we have

$$
Q \ll x^{-2 \eta} \min \left\{x M_{3}^{-1}, x^{-1 / 2} M_{3}^{5 / 2}, x^{1 / 4} M_{3}^{3 / 4}, x^{-1 / 2} M_{3}^{7 / 2}\right\},
$$

which simplifies into

$$
Q \ll x^{-2 \eta} \min \left\{x M_{3}^{-1}, x^{-1 / 2} M_{3}^{5 / 2}, x^{1 / 4} M_{3}^{3 / 4}\right\},
$$

since we have $M_{3}>x^{1 / 3} \mathcal{L}^{-B / 3}$.

This holds by assumption in the setting of Proposition 6.2, with $\eta$ replaced by $\eta / 2$. After applying Proposition 7.3 (noting that $|f(r)| \leqslant\left|F_{3}(\mathrm{i} t)\right| \ll 1$ and $\left.\sup _{r \sim R}\left|f^{\prime}(r)\right| \ll T\right)$ we derive

$$
\begin{aligned}
\Sigma_{0}(Q, \boldsymbol{M}, 1)= & \frac{1}{2 \pi \mathrm{i}} \int_{-\mathrm{i} T}^{\mathrm{i} T} F_{3}(\mathrm{i} t) \sum_{m_{1}} \sum_{m_{2}} \frac{\left(V_{1}\left(m_{1}\right) m_{1}^{\mathrm{i} t}\right) \cdot\left(V_{2}\left(m_{2}\right) m_{2}^{\mathrm{i} t}\right)}{\varphi\left(m_{1} m_{2}\right)} \\
& \times \sum_{\substack{q \sim Q, r \\
\left(q r, m_{1} m_{2}\right)=1}} \sum_{q}\left(c_{q} q^{-\mathrm{i} t}\right) \cdot r^{-\mathrm{i} t} d t+O\left(x^{1-\eta_{1}+2 v}\right)
\end{aligned}
$$

where $\eta_{1}>0$ depends on $\eta$.

Using the Mellin inversion formula again, we then deduce

$$
\begin{aligned}
\Sigma_{0}(Q, \boldsymbol{M}, 1)= & \sum_{m_{1}} \sum_{m_{2}} V_{1}\left(m_{1}\right) V_{2}\left(m_{2}\right) \sum_{\substack{q \sim Q, r \\
\left(q r, m_{1} m_{2}\right)=1}} \frac{c_{q}}{\varphi\left(m_{1} m_{2}\right)} V_{3}\left(\frac{q r}{m_{1} m_{2}}\right) \\
& +O\left(x^{1-\eta_{1}+2 v}\right) .
\end{aligned}
$$

Next, from Lemma 7.2, we get

$$
\sum_{\substack{r \\\left(r, m_{1} m_{2}\right)=1}} V_{3}\left(\frac{q r}{m_{1} m_{2}}\right)=\frac{\varphi\left(m_{1} m_{2}\right)}{q} \cdot \widehat{V}_{3}(0)+O\left(d\left(m_{1} m_{2}\right) \mathcal{L}^{2 B}\right),
$$


and hence finally

$$
\begin{aligned}
\Sigma_{0}(Q, \boldsymbol{M}, 1)= & \sum_{m_{1}} \sum_{m_{2}} V_{1}\left(m_{1}\right) V_{2}\left(m_{2}\right) \sum_{\substack{q \sim Q \\
\left(q, m_{1} m_{2}\right)=1}} \frac{c_{q}}{q} \widehat{V}_{3}(0) \\
& +O\left(x^{1-\eta_{1}+2 v}\right)+O\left(Q \mathcal{L}^{2 B+2}\right) \\
= & \sum_{m_{1}} \sum_{m_{2}} V_{1}\left(m_{1}\right) V_{2}\left(m_{2}\right) \sum_{\substack{q \sim Q \\
\left(q, m_{1} m_{2}\right)=1}} \frac{c_{q}}{q} \widehat{V}_{3}(0)+O\left(x^{1-\eta_{1}+2 v}\right),
\end{aligned}
$$

(if we assume $\eta_{1}<1 / 4$, which we can certainly do).

We are now almost done, but before performing the last steps, we will generalize this formula to an arbitrary integer $a \neq 0$. The reader may skip the next section in a first reading.

7.3. The case of general $a$. We will generalize (7.11) in this section to the sum $\Sigma_{0}(Q, \boldsymbol{M}, a)$ for a non-zero fixed integer $a$.

For an arbitrary arithmetic function $f\left(m_{1}, m_{2}\right)$ with bounded support, we have the decomposition

$$
\sum_{m_{1}} \sum_{m_{2}} f\left(m_{1}, m_{2}\right)=\sum_{\delta \mid a} \sum_{\delta=\delta_{1} \delta_{2}} \sum_{\substack{\delta_{1} \mid m_{1} \\\left(\frac{m_{1}}{\delta_{1}}, \frac{a}{\delta_{1}}\right)=1}} \sum_{\substack{\delta_{2} \mid m_{2} \\\left(\frac{m_{2}}{\delta_{2}}, \frac{a}{\delta}\right)=1}} f\left(m_{1}, m_{2}\right)
$$

(put $\left.\delta_{1}=\left(a, m_{1}\right), \delta_{2}=\left(a / \delta_{1}, m_{2}\right)\right)$. We apply this formula to

$$
f\left(m_{1}, m_{2}\right)=\sum_{\substack{q \sim Q, r \\ q r \equiv-a \bmod m_{1} m_{2}}} c_{q} V_{3}\left(\frac{q r+a}{m_{1} m_{2}}\right) .
$$

Starting from the analogue of (7.4) for an arbitrary $a$, we define $a^{\prime}=a / \delta$, $m_{1}^{\prime}=m_{1} / \delta_{1}, m_{2}^{\prime}=m_{2} / \delta_{2}$ and $r^{\prime}=r / \delta$ and split the congruence (7.3) into $O_{a}(1)$ sums corresponding to the congruences $q r^{\prime} \equiv a^{\prime} \bmod m_{1}^{\prime} m_{2}^{\prime}$, where now we have $\left(m_{1}^{\prime} m_{2}^{\prime}, a^{\prime}\right)=1$ (recall also that $c_{q}=0$ when $a$ and $q$ are not coprime). Hence proceeding as before, the formula (7.11) generalizes to

$$
\begin{aligned}
\Sigma_{0}(Q, \boldsymbol{M}, a)= & \sum_{\delta \mid a} \sum_{\delta=\delta_{1} \delta_{2}} \sum_{\left(m_{1}^{\prime}, a / \delta_{1}\right)=1} V_{1}\left(\delta_{1} m_{1}^{\prime}\right) \\
& \times \sum_{\left(m_{2}^{\prime}, a / \delta\right)=1} V_{2}\left(\delta_{2} m_{2}^{\prime}\right) \sum_{\substack{q \sim Q \\
\left(q, m_{1}^{\prime} m_{2}^{\prime}\right)=1}} \frac{c_{q}}{q} \widehat{V}_{3}(0)+O\left(x^{1-\eta_{1}+2 v}\right)
\end{aligned}
$$

for any fixed integer $a \neq 0$. When $a=1$, this formula becomes simply (7.11). We thus can continue with it in the general case. 
7.4. End of the proof. In equation (7.12), we now exchange the order of the sums, and apply Lemma 7.2 again to deal with the sums over $m_{1}^{\prime}$ (coprime with $a q / \delta_{1}$ ) and $m_{2}^{\prime}$ (coprime with $a q / \delta$ ). By the assumption (4.11) and the bound $M_{1} \leqslant M_{2} \leqslant M_{3} \leqslant x^{11 / 23}$, the variables $M_{1}$ and $M_{2}$ are not too small: we have

$$
M_{2} \geqslant M_{1} \geqslant \frac{x \mathcal{L}^{O(1)}}{M_{2} M_{3}} \geqslant x^{1 / 25}
$$

Therefore we have

$$
\begin{aligned}
\Sigma_{0}(Q, \boldsymbol{M}, a)= & \widehat{V}_{1}(0) \widehat{V}_{2}(0) \widehat{V}_{3}(0) \sum_{q \sim Q} \frac{c_{q}}{q} \sum_{\delta \mid a} \sum_{\delta=\delta_{1} \delta_{2}}\left(\frac{\varphi\left(\left(a / \delta_{1}\right) q\right)}{\left(a / \delta_{1}\right) q} \cdot \frac{1}{\delta_{1}}\right) \\
& \times\left(\frac{\varphi((a / \delta) q)}{(a / \delta) q} \cdot \frac{1}{\delta_{2}}\right)+O\left(x^{1-\eta_{1}+2 v}\right),
\end{aligned}
$$

provided that (say) $\eta_{1} \leqslant 1 / 1000$. The sum over $q$ is restricted to moduli coprime with $a$, and hence writing $a=\delta_{1} \delta_{2} \delta_{3}$, we find that the main term of the above expression is

$$
\widehat{V}_{1}(0) \widehat{V}_{2}(0) \widehat{V}_{3}(0) \sum_{q \sim Q} \frac{c_{q}}{q} \cdot\left(\frac{\varphi(q)}{q}\right)^{2} \frac{1}{a} \sum_{a=\delta_{1} \delta_{2} \delta_{3}} \sum_{\frac{\varphi\left(\delta_{2} \delta_{3}\right) \varphi\left(\delta_{3}\right)}{\delta_{2} \delta_{3}}} .
$$

Now, an elementary computation gives

$$
\sum \sum_{a=\delta_{1} \delta_{2} \delta_{3}} \sum_{\frac{\varphi\left(\delta_{2} \delta_{3}\right) \varphi\left(\delta_{3}\right)}{\delta_{2} \delta_{3}}}=\sum_{d \mid a} \frac{\varphi(d)}{d} \sum_{\delta \mid d} \varphi(\delta)=a,
$$

and therefore we finally get

$$
\Sigma_{0}(Q, \boldsymbol{M}, a)=\widehat{V}_{1}(0) \widehat{V}_{2}(0) \widehat{V}_{3}(0) \sum_{q \sim Q} \frac{c_{q}}{q} \cdot\left(\frac{\varphi(q)}{q}\right)^{2}+O\left(x^{1-\eta_{1}+2 v}\right) .
$$

Now gather (7.1), (7.14) and Lemma 7.1. The main terms disappear, and therefore

$$
\Sigma(Q, \boldsymbol{M}, a)=O\left(x^{1-\eta_{1}+2 v}\right),
$$

by (7.13), provided that (7.10) is satisfied. Now picking $v$ small enough, we obtain Proposition 6.2, which completes the proof of Theorem 1.2.

Acknowledgements. É. F. thanks ETH Zürich and EPF Lausanne for financial support.

\section{References}

1. E. Bombieri, J. Friedlander and H. Iwaniec, Primes in arithmetic progressions to large moduli. Acta Math. 156 (1985), 203-251.

2. E. Bombieri, J. Friedlander and H. Iwaniec, Primes in arithmetic progressions to large moduli. II. Math. Ann. 277(3) (1987), 361-393.

3. J.-M. Deshouillers and H. Iwaniec, Kloosterman sums and Fourier coefficients of cusp forms. Invent. Math. 70 (1982/1983), 219-288.

4. É. Fouvry, Autour du théorème de Bombieri-Vinogradov. Acta Math. 152(3-4) (1984), 219-244. 
5. É. Fouvry, Sur le problème des diviseurs de Titchmarsh. J. Reine Angew. Math. 357 (1985), 51-76.

6. É. Fouvry and H. Iwaniec, Primes in arithmetic progressions. Acta Arith. 42(2) (1983), 197-218.

7. É. Fouvry, Ph. Michel and E. Kowalski, Algebraic twists of modular forms and Hecke orbits. Preprint, 2012, arXiv: 1207.0617.

8. É. Fouvry, Ph. Michel and E. Kowalski, Algebraic trace functions over the primes. Duke Math. J. (to appear); arXiv:1211.6043.

9. J. B. Friedlander and H. Iwaniec, Incomplete Kloosterman sums and a divisor problem (with an appendix by B. J. Birch and E. Bombieri). Ann. of Math. (2) 121(2) (1985), 319-350.

10. D. R. Heath-Brown, The divisor function $d_{3}(n)$ in arithmetic progressions. Acta Arith. 47 (1986), $29-56$.

11. H. Iwaniec and E. Kowalski, Analytic Number Theory (American Mathematical Society Colloquium Publications 53), American Mathematical Society (Providence, RI, 2004).

12. Y. Motohashi, An induction principle for the generalization of Bombieri's prime number theorem. Proc. Japan Acad. 52(6) (1976), 273-275.

13. D. H. J. Polymath, New equidistribution estimates of Zhang type, and bounded gaps between primes. Preprint, 2014, arXiv: 1402.0811.

14. P. Shiu, A Brun-Titchmarsh theorem for multiplicative functions. J. Reine Angew. Math. 313 (1980), 161-170.

15. D. Wolke, Über die mittlere Verteilung der Werte zahlentheoretischer Funktionen auf Restklassen, I. Math. Ann. 202 (1973), 1-25.

16. Y. Zhang, Bounded gaps between primes. Ann. of Math. (2) 179(3) (2014), 1121-1174; doi: 10.4007/annals.2014.179.3.7.

Étienne Fouvry,

Université Paris Sud,

Laboratoire de Mathématique,

Campus d'Orsay,

91405 Orsay Cedex,

France

E-mail: etienne.fouvry@math.u-psud.fr

Philippe Michel,

EPFL/SB/IMB/TAN, Station 8,

CH-1015 Lausanne,

Switzerland

E-mail: philippe.michel@epfl.ch
Emmanuel Kowalski,

ETH Zürich - D-MATH,

Rämistrasse 101,

CH-8092 Zürich,

Switzerland

E-mail: kowalski@math.ethz.ch 